Teknokultura. Revista de Cultura Digital y Movimientos Sociales

ISSNe: $1549-2230$

http://dx.doi.org/10.5209/TEKN.62054

\title{
Lógicas de funcionamento do Tinder. Uma análise da aplicação e das perceções dos utilizadores ${ }^{1}$
}

Recibido: 23 de octubre de 2018 / Aceptado: 15 de marzo de 2019 Open peer reviews

\author{
Rita Sepúlveda²; Jorge Vieira ${ }^{3}$
}

Resumo. O número de aplicações de mobile dating tem vindo a aumentar, mas o Tinder goza de um reconhecimento por parte dos utilizadores e de uma visibilidade mediática e estudo científico que o distingue das demais. Porém, ainda não é claro como os diferentes agentes em jogo interagem, nem como as informações dos utilizadores são tratadas e combinadas através da ação dos algoritmos. Na procura de elucidar esses aspetos, este artigo explora como o design, arquitetura e affordances da plataforma estruturam as ações dos utilizadores e, consequentemente, moldam a utilização, questionando de seguida, como as informações e utilização geram determinados resultados através da ação do algoritmo. Para tal, recorreu-se a uma metodologia qualitativa, combinando duas técnicas de recolha de dados o que permitiu, por um lado, uma análise crítica através da identificação das diferentes possibilidades de funcionamento do Tinder e de como é imposto um fluxo de utilização e, por outro, através dos discursos dos utilizadores, compreender como estes interpretam as affordances da aplicação no decorrer da sua utilização. Como principais resultados, verificou-se que parte dos utilizadores não parecia estar consciente da presença e ação do algoritmo. Indicaram também que as suas ações estavam condicionadas em diversos momentos da utilização, porém contornavam (alguns) (d)esses condicionantes encontrando novas formas de uso.

Palavras-chave: affordances; algoritmo; app; online dating.

\section{[en] Tinder's operating logics. An analysis of the application and the perceptions of the users}

\begin{abstract}
The number of mobile dating apps has been increasing. Among them, Tinder has achieved user recognition and received attention from the press and academia. However, it is still not clear how the different agents involved interact, nor how user information is processed and combined through the action of algorithms, shaping the results of using the app. Endeavoring to shed some light on these aspects, this paper explores how the design, architecture and affordances of the platform structure user actions and consequently shape the method of use questioning how the information is used and how the actions performed generate certain results through the action of algorithms. To do so, a qualitative methodology was used combining two data collection techniques: walkthrough method and interviews. This enables on the one hand a critical analysis to be made, by identifying Tinder's different ways of working and how the usage flow is imposed; and on the other hand, understanding through users' discourse, how the latter interprets the app's different affordances while using it. The main results show that users indicated that their actions were constrained at various times during use, although they circumvented (some) of the constraints by finding new methods of use. Some of the users did not seem to be aware of the presence and action of the algorithm, nor of how their information was used.
\end{abstract}

1 Este trabalho foi financiado através de fundos do MCTES / FCT - Fundação para a Ciência e a Tecnologia, no âmbito da bolsa de doutoramento com a referência SFRH/BD/130648/2017.

2 Instituto Universitário de Lisboa (ISCTE-IUL), Centro de Investigação e Estudos de Sociologia (CIES-IUL), Lisboa, Portugal. E-mail: racfc@iscte-iul.pt

3 Instituto Universitário de Lisboa (ISCTE-IUL), Centro de Investigação e Estudos de Sociologia (CIES-IUL), Lisboa, Portugal. E-mail: jorge_vieira@iscte-iul.pt 
Keywords: affordances; algorithm; app; online dating.

Sumário. 1. Introdução. 2. Tinder: caracterização. 3. Tinder: affordances e algoritmos. 4. Metodologia. 5. Recrutamento e corpus de análise. 6. Caracterização da amostra. 7. Resultados e discussão. 7.a. Registo. 7.b. Perfil. 7.c. Definições. 7.d. Utilização. 8. Considerações finais. 9. Agradecimentos. 10. Referências.

Cómo citar: Sepúlveda, R. y Vieira, J. (2019). Lógicas de funcionamento do Tinder. Uma análise da aplicação e das perceções dos utilizadores, en Teknokultura. Revista de Cultura Digital y Movimientos Sociales, 16(1), 75-91.

\section{Introdução}

Encontram-se disponíveis um crescente número de plataformas e aplicações de online dating (Light, 2014). Nos últimos anos estas têm vindo a registar um crescimento na oferta e procura (Albury, Burgess, Light, Race e Wilken, 2017), sendo que entre as aplicações sociais, são aquelas com maior número de downloads na App Store em todo o mundo (Online dating, 2018). Tal cria novas dinâmicas na área dos relacionamentos sociais, na medida em que ao desenvolverem-se através de aparelhos móveis podem gerar diferentes discursos ou dinâmicas de género ou sexuais (Goluboff, 2015) diferenciados.

Com o aumento da oferta de aplicações de online dating, aumentou também a atenção conferida às mesmas tanto pelos media como pela academia, onde um número crescente de investigadores focaram o seu trabalho em questões tais como: autenticidade (Duguay, 2017), autoapresentação (Ellison, Heino e Gibbs, 2006; Casimiro, 2014; Vieira, 2017), influência no sexo casual (Choi, Wong, Lo, Wong, Chio e Fong, 2016; Licoppe, Riviére e Morel, 2016) ou motivações (Sumter, Vandenbosch e Ligtenberg, 2016; Timmermans e De Caluwé, 2017; Ranzini e Lutz, 2017).

Contudo, para além destas agendas de investigação, para compreender as aplicações sociais de online dating de forma holística é necessário conferir igual atenção às suas affordances (Gibson, 1979), dinâmicas de regulação (Gillespie, 2017), e lógicas de funcionamento, nomeadamente ao tratamento e combinação das informações através de algoritmos. Note-se que estas ações, para além de complexas, são opacas, ocorrendo nos bastidores da aplicação e, como tal, são desconhecidas e/ou esquecidas por parte dos utilizadores (Gillespie, 2014).

Com esta lacuna académica sinalizada e com este manifesto de investigação, definiu-se como pergunta de partida para a investigação: "De que forma a lógica de funcionamento do Tinder é percecionada pelos seus utilizadores?". O objetivo principal foi compreender como estes entendem a lógica de operabilidade interna da aplicação e o seu conjunto de funcionalidades e se estes parecem reconhecer o impacto na mediação e, como tal, no moldar de resultados.

Para tal, recorreu-se a metodologia qualitativa através do walkthrough method (Light, Burgess e Duguay, 2018; Duguay, 2017) e da realização de entrevistas, com o objetivo de identificar através dos discursos, mecanismos de funcionamento do Tinder e narrativas de descrição da utilização individual e perceções sobre este. 


\section{Tinder: caracterização}

O Tinder surgiu para smartphone em 2012 autoapresentando-se como a maior comunidade de solteiros do mundo, um site de encontros para conhecer pessoas novas ("What is Tinder", n.d.). Está disponível tanto para Android como para iOS sendo o seu download possível através das respetivas lojas onde está classificada como uma aplicação de "Estilo de Vida". Em 2017 foi disponibilizada uma versão web.

A utilização requer um perfil online, que pode ser criado através de uma conta de Facebook ou de um número de telefone, e funciona por geolocalização. Após a definição dos parâmetros de procura, são apresentados um conjunto de perfis e a lógica de utilização baseia-se em aceitar ou descartar perfis, através de um movimento de polegar (swipe). Após uma aceitação mútua - match-os utilizadores podem comunicar entre si através do sistema de mensagens interno.

O logotipo (chama), a forma como os perfis são criados e apresentados e a lógica de funcionamento: aceitar ou descartar perfis com base em informação limitada sem poder comunicar com o utilizador, levou a que fosse classificada como uma aplicação "de engate" (Sumter et al., 2016; LeFebvre, 2017; Sepúlveda, 2018)

É uma aplicação freemium na medida em que o uso é gratuito, mas através de uma paywall é restringido o acesso a um conjunto de funções apenas disponível após pagamento. Sucintamente, oferece na versão gratuita ações limitadas, onde os utilizadores podem ver um número restrito de perfis por dia, conversar, estando a distância de procura limitada a $160 \mathrm{~km}$. Têm acesso a um "Super like" diário (ícone estrela azul) cuja função permite indicar especial interesse por determinado perfil e cuja informação está disponível antes do utilizador aceitar ou rejeitar.

Existem opções pagas com diferentes planos mensais: Tinder Plus, Tinder Gold e micro pagamentos. Na versão Plus o utilizador pode retroceder na decisão de descartar um perfil (ícone seta desfazer), obter swipes ilimitados por dia, destacar uma vez por mês o seu perfil através da função "Boost" (ícone raio), obter cinco "Super Like" por dia, e aceder à função passaporte (ícone ponto de localização) através da qual não existem limitações de distância. A versão Tinder Gold engloba todas as possibilidades da versão Plus e acrescenta a possibilidade de saber que utilizadores deslizaram o perfil para a direita.

Estão disponíveis micro pagamentos para a funcionalidade Tinder "Boost" sendo que, segundo o Tinder (2016), esta característica aumenta a possibilidade de conseguir matches.

Entre as formas de receita do Tinder estão as opções pagas estimando-se, com base em estudos realizados e na ausência de dados divulgados pela própria empresa, que 24\% dos utilizadores mundiais subscrevem um pacote mensal (McGrath, 2015) e a oportunidade de marcas se publicitarem na aplicação ("Digital", n.d.). Porém, um utilizador individual não pode fazer qualquer tipo de publicidade ou promoção arriscando-se a que o seu perfil seja eliminado.

\section{Tinder: affordances e algoritmos}

Cada serviço de online dating é caracterizado por um conjunto de affordances, as quais, do ponto de vista da mediação e pela influência, podem ser condicionantes dos resultados. De acordo com Gibson (1979) as affordances podem ser descritas 
como a perceção individual e subjetiva, porém, baseada em características materiais objetivas que o utilizador tem sobre determinado objeto e o reconhecimento da sua usabilidade. Como tal, o resultado da interpretação das affordances de um mesmo objeto pode levar a formas de uso distintas por diferentes utilizadores.

Schrock (2015) identifica as quatro principais affordances comunicacionais comuns aos media móveis: a portabilidade, a disponibilidade, a localização e a multimédia. $\mathrm{O}$ Tinder, devido às suas características próprias, acrescenta a mobilidade $\mathrm{e}$ dominância visual (Chan, 2017).

A mobilidade vai além da portabilidade, ao se poder utilizar a aplicação em qualquer espaço, seja este público ou privado (Chan, 2017) e ainda com um propósito de entretenimento, navegando através dos perfis (Ranzini e Lutz, 2017). A disponibilidade remete para o sistema de notificações que alerta quando há um novo match ou uma mensagem, sendo possível ver ou responder no momento, uma vez que os utilizadores normalmente levam o smartphone consigo (Yeo e Fung, 2016). A affordance localização está relacionada com o funcionamento por geolocalização, classificando o Tinder como uma aplicação location-based real time dating (LBRTD) remetendo para aspetos como a proximidade ou a imediatez (Hjorth, 2012; Duguay, 2017), na medida em que através do GPS do smartphone informa sobre o posicionamento, partilhando-o com outros (Blackwell, Birnholtz e Abbott, 2014) e podendo proporcionar o encontro (Duguay, 2017). Por fim, a affordance multimédia está relacionada com a criação do perfil, com recurso a imagens e textos, através do Facebook, e a integração de outras plataformas como o Spotify ou Instagram. Ranzini e Lutz (2017) argumentam que a affordance multimédia é limitativa devido à estruturação das formas de apresentação dos perfis aos utilizadores: uma foto principal, nome e idade, deslizando estes conforme o seu interesse (David e Cambre, 2016) e nem sempre visualizando mais informação sobre o utilizador.

Para além da arquitetura do Tinder, que em grande parte se reflete nas affordances, são incorporados na aplicação algoritmos, caracterizados pela sua natureza pouco clara (Gillespie, 2017; Courtois e Timmermans, 2018), influindo na experiência e podendo determinar o sucesso de um perfil. Esta é baseada na informação fornecida no momento da criação do perfil, mas também na utilização da aplicação, sendo que através da análise em tempo real dos dados, o algoritmo procura aumentar não só a experiência de utilização, mas também o desempenho da aplicação (Alpaydin, 2014).

Autores defendem que, através da ação do algoritmo, é determinado o resultado que os proprietários da plataforma desejam, entre os quais, se encontram o recurso a opção pagas de forma a maximizar o lucro (Domingos, 2017). Ou seja, um dos objetivos da ação do algoritmo é conseguir manter os utilizadores da versão gratuita relativamente satisfeitos, de forma a que não se sintam frustrados na utilização abandonando a plataforma, mas também, em certos momentos, insatisfeitos e querendo algo mais, de forma a recorrerem às opções pagas (Courtois e Timmermans, 2018).

Para tal, o fluxo contínuo de dados do utilizador serve como recurso de aprendizagem dos algoritmos. Assim, a experiência do utilizador está condicionada pelos proprietários das plataformas, na medida em que estes determinam a arquitetura, construindo os seus discursos (van Dijck, 2013), mas também pela ação dos programadores através de protocolos, configurações e construção de algoritmos que pela sua forma semiautónoma de aprendizagem, pretendem melhorar a experiência para 
que os utilizadores permaneçam ativos, pois enquanto ativos vão fornecendo dados, mas também de forma a determinar o potencial momento ideal para converter a utilização gratuita em paga. Todos estes elementos terão influência nos resultados da utilização de determinada plataforma (van Dijck, 2013; Gillespie, 2017; Courtois e Timmermans, 2018).

\section{Metodologia}

Para compreender os discursos dos utilizadores, suas perceções e literacias digitais foi necessário num primeiro momento tomar contacto direto com a aplicação, explorando as suas affordances e fluxo de funcionamento. Para tal recorreu-se sequencialmente a dois métodos qualitativos de recolha de dados, procedendo-se posteriormente à análise dos dados gerados.

Através do walkthrough method, técnica utilizada para a análise de aplicações, foi possível o contacto direto com o Tinder por parte dos investigadores como se de utilizadores se tratassem e com o objetivo de se obter um conhecimento empírico da aplicação. Para tal, procedeu-se ao registo no Tinder em dezembro de 2017, tendo o perfil sido criado através do Facebook. A análise foi realizada com a versão gratuita do Tinder, instalada num iPhone 7 , com o sistema operativo iOS11 e em Portugal ${ }^{4}$.

Após informados de aspetos como a dinâmica de funcionamento, fluxo e possibilidades na utilização, foram realizadas entrevistas semiestruturadas a utilizadores ativos do Tinder. O guião das mesmas era composto por duas dimensões analíticas: 1. Registo e criação do perfil, explorando como procederam ao registo e dando atenção à informação partilhada; 2. Utilização, focando-se o uso e a interação com os utilizadores.

Com o recurso a entrevistas semiestruturas e devido à sua flexibilidade foi possível recolher dados discursivos em função do decurso de cada conversa (Bryman, 2012), introduzindo questões de acordo com os inputs dos participantes. Estas foram realizadas face a face e, por a investigação incidir sobre a intimidade de cada um, acrescentámos a possibilidade de realizá-las por Skype, partindo do pressuposto de que estas poderiam ser uma boa solução, quando complementadas com as presenciais (Lo Iacono, 2016).

\section{Recrutamento e corpus de análise}

Os participantes foram recrutados através da criação de um perfil no Tinder (Blackwell et al., 2014; Ward, 2016), onde na informação visual como na textual se informava sobre a realização do estudo. Aliou-se o recrutamento através de um questionário online, que se estava a realizar no âmbito mais abrangente da investigação, e onde foi reservado um campo onde os interessados podiam facultar os seus dados de contacto para serem entrevistados. Por fim, colocou-se uma entrada no site Reddit.

No total foram realizadas 18 entrevistas, entre dezembro de 2017 e setembro de 2018 com uma duração entre 20 e 88 minutos. Após transcritas foram analisadas

4 Consideramos importante referir a localização, já que tal informação é tida em consideração pelo algoritmo existindo determinadas funcionalidades disponíveis apenas em certas localizações. 
codificando-se a informação gerada, o chamado elicited data (Salmons, 2016). No processo de codificação os dados gerados foram interpretados de acordo com conceitos teóricos precedentes sendo as categorias criadas com origem nestes, mas também nos dados resultantes da análise, referidos como concept driven coding e data driven coding (Krippendorff, 2012) respetivamente.

\section{Caracterização da amostra}

A amostra, por conveniência, foi composta por 18 utilizadores, com idades compreendidas entre os 19 e os 54 anos, registados no Tinder entre 6 meses e 5 anos e de nacionalidade Portuguesa. Entre os 18 entrevistados, 13 identificaram-se como sendo do género masculino e 5 do género feminino. Apesar do género masculino estar sobre representado, tal facto não interfere na análise de resultados uma vez que não se pretendem realizar estudos comparativos de natureza de género nem este será aqui considerado como variável de análise.

Os participantes caracterizavam-se por, na sua maioria, terem formação superior $(n=15)$, estando a trabalhar $(n=12)$, a estudar $(n=4)$ ou em situação de desemprego $(n=2) .17$ entrevistados indicaram que eram heterossexuais e 1 homossexual. Quanto ao estado civil, 4 indicaram que estavam divorciados e 14 solteiros. Por fim, de entre os 18 participantes, apenas 1 indicou que utilizava uma versão paga da aplicação.

\section{Resultados e discussão}

\subsection{Registo}

Após download do Tinder surgiu uma sequência de quatro ecrãs que se ilustram na figura 1 , sendo metade ocupado por uma imagem e a respetiva legenda, resumindo a lógica de utilização. A primeira tratava-se da imagem de uma suposta utilizadora (branca, de cabelos loiros, com roupa informal e sorridente) e com a legenda "descubra pessoas novas e interessantes perto de si" indicando o propósito de funcionamento e remetendo para o funcionamento por geolocalização. A segunda imagem era uma reprodução da primeira à qual se acrescentou a palavra "Like" a verde e que remete para a ação a realizar: se gostar, deslize a foto para a direita, se não gostar, deslize a foto para a esquerda. Na terceira imagem surgem dois perfis, a suposta utilizadora e um suposto utilizador (negro, sorridente e com óculos) acompanhados da expressão "It's a Match", reproduzindo o fluxo de uso, após criar perfil, visualizam-se os outros e desliza-se para a direita os que se gostam aumentando a possibilidade de correspondência. Nesta terceira imagem o Tinder reflete uma postura hétero normativa (Duguay, 2017) legitimando e privilegiando os relacionamentos heterossexuais, mas também para remetendo para um pluralismo de pertenças étnicas entre os seus utilizadores. Na última imagem reproduz-se o que seria o chat do Tinder, informando que a comunicação com outros apenas é possível após a existência de correspondência - característica distintiva da aplicação. 
Figura 1. Sequência de 4 ecrãs correspondente ao processo de registo do Tinder. Fonte: App Tinder. Consultado dezembro 2017.
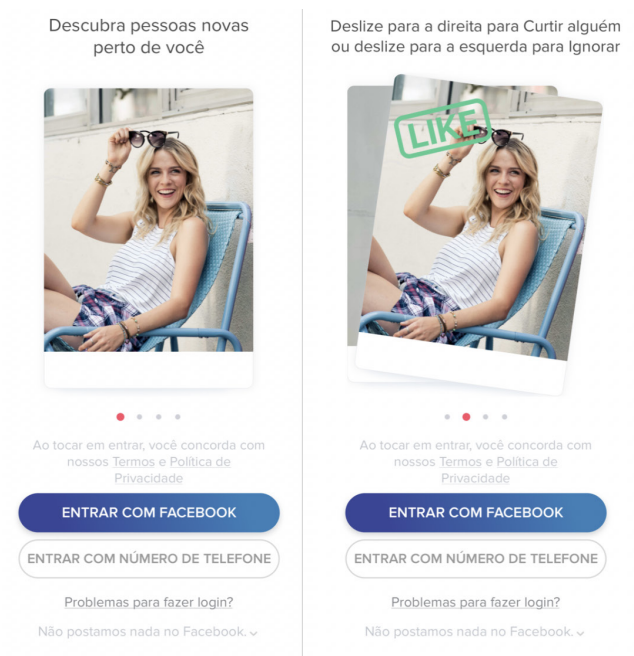

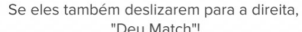
"Deu Match"!
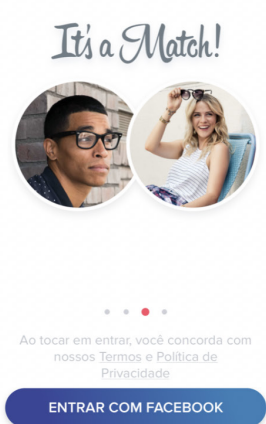

ENTRAR COM NÚMERO DE TELEFONE

Problemas para fazer login?
Só os seus Matches podem conversar com você

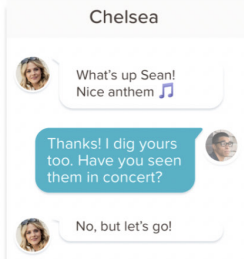

ENTRAR COM FACEBOOK

ENTRAR COM NÚMERO DE TELEFONE

Problemas para fazer login?

Na parte inferior dessas imagens, surgiram as duas opções de como efetuar o registo: através do Facebook, carregando num botão visualmente destacado de cor azul chamando à atenção do utilizador, ou através do número de telemóvel, cuja opção aparece sem qualquer destaque. Independentemente da opção escolhida, os termos e serviços de uso são aceites automaticamente.

Ao iniciar a sessão com o Facebook, o Tinder permite fazê-lo através da aplicação da rede social ou abrindo o browser. Nesse momento, o utilizador é forçado a tomar uma decisão pois a forma como o Tinder está desenhado não permite utilização, nem que seja apenas para experimentação, sem registo e consequente criação de perfil.

Neste contexto, os entrevistados referiram que após terem conhecimento do Tinder, normalmente através de amigos ou dos media, acabavam por instalar e "compulsivamente" começar a usar, ainda que de forma mais experimental ou para satisfazer a curiosidade. Porém, a forma como era efetuado o registo e consequente ligação ao Facebook nem sempre era clara para os entrevistados: "Descarreguei e automaticamente as minhas informações foram para lá, eu acho que devem ter ido do Facebook" (Entrevistado, 14)

\subsection{O perfil}

O registo no Tinder é sinónimo de criação de um perfil. Fazendo-o através da aplicação Facebook, versão mais comum entre os entrevistados $(n=18)$ e única disponível entre 2012 e julho de 2017, surge uma mensagem no ecrã que informa que serão importados dados como a lista de amigos, a data de nascimento, fotografias, gostos e endereço de e-mail, informando que a edição dos dados é possível e apesar da ligação entre as duas redes, o Facebook não permite que o Tinder publique no mesmo remetendo para questões de privacidade.

O perfil é composto por nome, idade, género, um limite de seis fotografias, escola, trabalho, campo "sobre mim" -espaço de 500 caracteres onde o utilizador pode 
completar a sua biografia, e interesses- páginas do Facebook às quais o utilizador colocou "gosto". Os interesses serão apresentados em caso de serem comuns com um potencial match. Caso o utilizador deseje, pode editar informações, clicando no respetivo ícone - lápis, ainda que algumas delas como nome, idade, escola e trabalho, seja necessário editá-las primeiro no Facebook, como forma de confirmação.

É possível alterar o género sendo que, as opções estão restringidas a homem ou mulher. Apesar do Facebook conferir a opção de personalização do género, no Tinder só os dois referidos estavam disponíveis, limitando a diversidade, reproduzindo padrões e impondo a norma social.

O processo conclui-se ao clicar no botão "continuar" sendo o perfil criado de forma automática. A possibilidade de criar o perfil através do Facebook remete tanto para questões de "convergenceability" (Marcus, 2016) que consiste no pouco esforço que os utilizadores têm que investir na criação de um perfil comparativamente com outras redes de dating, como para questões de autenticidade (Duguay, 2017), porém não a garante. Três participantes referiram terem-se sentido incomodados com o facto de ao se encontrarem pessoalmente com outros utilizadores, verificarem que esses não se assemelhavam às fotografias, remetendo para questões de engano e desilusão na apresentação (Guadagno, 2012; Ellison et al., 2006; Gibbs, Ellison e Heino, 2006).

Outro utilizador partilhou ter sido alvo de tentativa de um esquema piramidal e outro referiu que no decorrer de um encontro a utilizadora revelou-lhe ser prostituta e caso ele estivesse interessado teria de pagar. Estas situações remetem para o conceito de catfish que consiste, em contexto de online dating, de forma intencional, usar uma identidade falsa no sentido de se envolver emocionalmente ou romanticamente com outrem (Schiling, n.d.). No discurso de um dos utilizadores o termo estava inclusivamente presente: "Sim, acho que que já me aconteceu ser catfish, mas acho que isso a quem é que não aconteceu. Não é? Por acaso não muitas vezes (...) é um bocado awkward..." (Entrevistado, 11)

O Tinder incentiva os utilizadores a reportarem situações com as quais se sentem incomodados. A denúncia, anónima, pode ser feita através da lista de matches, diretamente no perfil que pretende denunciar ou nas mensagens. No mesmo sentido, a construção e edição do perfil está condicionada a um conjunto de regras, sendo que o seu desrespeito, pode resultar na eliminação da fotografia ou na eliminação do perfil.

As fotografias podem ser eliminadas ou substituídas por outras do Facebook ou do álbum do dispositivo móvel, sendo que o Tinder evita perfis sem fotografias, não permitindo a possibilidade de apagar a totalidade das imagens e impondo regras referentes ao tipo de fotografias, condicionando assim a autoapresentação dos utilizadores. Três dessas regras mais evidentes são a restrição na partilha de fotografias de caça, na medida em que podem ferir suscetibilidades, a proibição de partilha de fotografias de crianças sozinhas, mesmo que seja uma fotografia do próprio, e a proibição de imagens com nudez, sexualmente explicitas ou inclusive de poses sugestivas. Esta regra tem como consequência que os utilizadores do género masculino e feminino sejam tratados de forma distinta, podendo os primeiros colocar no seu perfil fotografias em que estejam em tronco nu. No caso do género feminino são consideradas nudez e a fotografia é eliminada. Este condicionamento remete para uma reflexão sobre o que é convencionado como socialmente aceitável e em como as redes sociais reproduzem padrões normativos clássicos da sociedade (Gillespie, 
2017). O Tinder argumenta, nas suas regras de utilização, que o objetivo é manter a aplicação "elegante e apropriada para consumo público" ("Community", n.d.).

A função "fotografia inteligente", disponível para aqueles utilizadores que têm no seu perfil três ou mais fotografias, coloca em primeiro lugar aquela considerada pelo algoritmo como provável de ser mais gostada pelos utilizadores, aumentando assim a probabilidade de match (“Apresentamos", 2016). Esta função não foi referida por nenhum dos utilizadores durante as entrevistas.

$\mathrm{Na}$ construção e consequente apresentação dos perfis a atribuição da atenção por parte da aplicação à componente visual gerou uma posição crítica por parte dos utilizadores levando-os, no caso dos que já tinham experimentado outras aplicações $(\mathrm{n}=7)$, a indicar a simplicidade na criação de perfil no Tinder como uma vantagem face a estas, mas por outro lado a argumentar que o facto de não existirem perguntas no momento de criação do perfil limitava as possibilidades na apresentação e de possíveis matches, chegando a apelidar o Tinder como "superficial" (Entrevistado, 2), "abrasiva" (Entrevistado, 8) e "centrado na fotografia" (Entrevistado, 10).

No campo "Sobre Mim" a informação partilhada também está sujeita a regras proibindo os discursos violentos ou desrespeitosos assim como a partilha de contactos como o número de telefone, a morada ou outros dados como número de cartão de crédito. $\mathrm{O}$ Tinder argumenta que a sua lógica de funcionamento, com a aceitação mútua, resulta em que, caso o utilizador assim o deseje, possa partilhar determinada informação, mas apenas de forma privada.

Quando questionados os entrevistados sobre a construção do seu perfil e o conteúdo do mesmo, grande parte afirmou $(\mathrm{n}=13)$ que não tinha perdido muito tempo com o processo, não trocando fotografias frequentemente, admitindo que aquelas que tinham já estavam há muito tempo. Quanto à informação textual, a maioria indicou ter algo escrito $(\mathrm{n}=17)$, mas informação resumida.

Entre aqueles que no seu discurso indicaram refletir sobre a construção do perfil $(n=5)$, revelaram cuidado na escolha das fotografias, da adequação das fotografias à estação do ano, e que estas permitissem dar a conhecer os seus gostos ou atividades. Revelaram que o objetivo era que o perfil refletisse algo sobre si para quem o visse, remetendo para o conceito de utilizador curador (Hogan, 2010) decidindo que informação partilhar.

"Tentei descrever-me o melhor, usando as fotos para representar um pouco de mim." (Entrevistado, 9)

Por fim, é possível integrar no perfil contas de duas aplicações: Spotify e Instagram, aparecendo no perfil as músicas e as fotografias respetivamente. A integração do Instagram surgiu após a compra da rede pelo Facebook. Antes disso os utilizadores colocavam o respetivo nome de utilizador do Instagram no campo "Sobre mim" completando a sua autoapresentação (Prigg, 2015) e contornando as imposições colocadas pela aplicação. Os entrevistados que referiram ser interessante $(n=5)$, argumentavam que se devia ao facto de poderem ver mais fotografias, já que tinham acesso a um maior número, mas que servia também como forma de contornar o fechamento da arquitetura da aplicação, que apenas permite falar com outro quando existe match, já que ao estar o perfil de Instagram identificado, era possível procurá-lo e começar a seguir e chegar ao contacto.

Uma vez criado o perfil, a fotografia definida como principal ocupa o ecrã do dispositivo e em baixo surge o nome e a idade. Caso o utilizador tenha mais do que uma fotografia, aparece uma barra na parte superior, seccionada em função do número de 
fotografias. Para ver as restantes basta clicar no ecrã. No canto inferior direto surge o ícone "i”, ao clicar acede-se à restante informação partilhada em "Sobre mim" e interesses em comum. Esta lógica de apresentação e dinâmica de funcionamento, referida como um catálogo (David e Cambre, 2016), foi identificada no discurso de um entrevistado "Uma loucura, só na onda do catálogo. Passa para ali, passa para aqui, pronto.” (Entrevistado, 15).

\subsection{Definições}

Após a criação do perfil o utilizador deve estabelecer um conjunto de definições, inicialmente predefinidas de acordo com o que o algoritmo considerou indicado em função da informação partilhada. São eles a localização, o género e a idade.

O utilizador define a distância de procura num raio máximo de $160 \mathrm{~km}$. Porém, não significa que os resultados sejam semelhantes em qualquer região sendo as zonas urbanas privilegiadas (Duguay, 2017). Dois entrevistados referiram utilizar a aplicação frequentemente, independentemente do local onde se encontravam, mencionando a facilidade em encontrar outros utilizadores nomeadamente em zonas urbanas.

Autores argumentam que o funcionamento por geolocalização alimenta a intimidade móvel (Hjorth, 2012) assim como intensifica a imediatez e a possibilidade dos utilizadores se encontrarem (Duguay, 2017), contudo, ponto comum entre os participantes foi o de tal facto depender do momento em si, da pessoa em questão, de se lhes apetecia encontrar com outro utilizador ou não e da motivação pela qual se tinham registado. Dois utilizadores afirmaram inclusive que desde o registo não se tinham encontrado com outros e de entre os restantes 16, apenas um afirmou que recorria conscientemente à distância como opção de filtro.

Outro critério de procura a ser indicado é o género: "homens", "mulheres" ou "mulheres e homens". Estes são os únicos géneros possíveis traduzindo uma visão limitativa. O posicionamento do Tinder, como aplicação de engate, levou a que os utilizadores que desejavam conhecer outros de ambos os géneros fossem classificados como homossexuais ou alvos de abordagens indesejadas $(n=2)$, tal como relatou um participante: "Por muito incrível que pareça os homens são muito mais atiradiços que as mulheres e, portanto, não gostei das abordagens que tive pela parte de homens." (Entrevistado, 13)

O terceiro critério a definir é a faixa etária. A idade mínima são 18 anos e a máxima de 55 ou mais anos, não existindo distinção entre os utilizadores com idade superior. Segundo Duguay (2017), esta aglomeração entre as idades mais elevadas não é admirável já que a comunicação do Tinder está focada nos mais jovens uma vez que a maioria dos utilizadores tem idades compreendidas entre os 18 e os 34 anos (McGrath, 2015). Um entrevistado, consciente da utilização, referiu a importância da faixa etária como filtro, evitando fazer match com pessoas com idades inferiores ou superiores às das que pretendia conhecer.

\subsection{A utilizaçãa}

De acordo com os critérios estabelecidos, o algoritmo determina quem pode ver quem, apresentando aos utilizadores os seus potenciais matches (Courtois e Timmermans, 2018). O passo seguinte é aceitar ou descartar o perfil. Deslizando-o para a direita, ou carregando no ícone coração, o utilizador está a aceitá-lo, deslizando o 
perfil para a esquerda, ou carregando no ícone cruz, o utilizador está a descartá-lo e o perfil não volta, supostamente, a ser apresentado. $\mathrm{O}$ deslizar permanece anónimo entre os utilizadores, excetuando na utilização do "Super Like" e cujo propósito é impulsionar o match. Gillespie (2015) defende que, através desta lógica de utilização combinada com a ação do algoritmo, o Tinder promove uns utilizadores em detrimento de outros.

Porém, numa lógica de funcionamento que parece simples e exigindo um baixo nível de literacia digital, como referido por mais de metade dos entrevistados, nem sempre é percecionada de igual forma, como relata um deles: "Eu no início não percebia nada, não sabia como é que aquilo funcionava. O que é que era para a direita ou o que é que era para a esquerda." (Entrevistado, 14).

À referida lógica de funcionamento alia-se a relativa exigência de rapidez na tomada de decisão, se assim não for, não é possível visionar outros perfis (David e Cambre, 2016), levando inclusive a que os utilizadores desenvolvam novas lógicas de utilização como deslizarem os perfis sem verem as imagens ou lerem os textos que os compõem: "a maior parte das vezes não perco tempo a ver perfis ou a ver as descrições etc. ou seja, tudo swipe right e depois de dar match então sim, vou ver a pessoa" (Entrevistado, 13).

Esta utilização focada na maximização, apelidada de shotgun approach (LeFebvre, 2017), consiste em aceitar o maior número de perfis possíveis com o propósito de potencialmente aumentar a probabilidade de um maior número de matches, embora um maior número de matches não signifique um maior número de encontros (Courtois e Timmermans, 2018).

Não obstante, o número de swipes diários está limitado pela plataforma e quando o utilizador o atinge só poderá voltar a deslizar passadas 12 horas, podendo esta restrição incitar os utilizadores a optarem por versões pagas (O’Brien, 2015; Courtois e Timmermans, 2018). Não existem dados oficiais sobre o número de swipes disponíveis por dia, mas informações apontam que variam em função do perfil e utilização ("What is", 2018). Este fator foi apontado por um dos participantes como condicionante da sua utilização como descreve: "aquilo tem tipo um número limitado e depois precisa de horas e é do género já que estou a usar vou ser eficiente, mas é estranho porque é muito pouco lógico porque é do género eu tenho necessidade de gastar." (Entrevistado, 8).

Três participantes indicaram abertamente sentirem-se frustrados com os resultados da sua utilização: reduzido número de matches e não conversão destes em encontros. Explorando os seus perfis, indicaram não ter prestado grande atenção à construção do mesmo nem consideraram editá-lo. Ao nível da interação, dois participantes mencionaram que não conseguiram estabelecer conversas satisfatórias com outros utilizadores levando-os a deixarem de trocar mensagens, e entre aqueles com os quais conversavam indicavam que ainda não tinham conseguido concretizar a vontade de se encontrarem com os mesmos, sentindo-se rejeitados. Esta junção de fatores originou a que estivessem largos períodos sem utilizar a aplicação. Carr (2016) argumenta que o algoritmo do Tinder, classifica os utilizadores em função do seu grau de atração, diretamente ligado com o número de swipes, matches e com quem dão matches, revelando também que a inatividade prejudica o utilizador. Esta lógica foi apontada por um utilizador sendo consciente da ação ao algoritmo: "é muito claro, se eu parar de usar não tenho matches nenhuns porque não gosto de ninguém e a partir de uma certa altura o Tinder mostra-me como invisível." (Entrevistado, 11) 
A função unmatch está disponível, podendo acontecer unilateralmente garantindo que os perfis desses utilizadores não voltam a ser mutuamente sugeridos e o historial de conversação é eliminado. Alguns entrevistados $(n=5)$ indicaram recorrer a esta função em algumas situações, nomeadamente, nas quais se sentiam de alguma forma ofendidos, nas quais não tinham interesse em continuar em contacto, podendo acontecer após um encontro, ou quando exploravam o perfil e concluíam que não tinham interesse no mesmo.

Por fim e tratando-se de uma plataforma criada para a lógica mobile, sem restrições espaciais, são criadas novas dinâmicas de utilização deixando esta de ser uma prática privada e exclusivamente individual. Três participantes referiram utilizar a aplicação com amigos, deixando inclusive que estes decidissem por si. Esta prática foi apelidada por um dos participantes como "jogar ao Tinder" (Entrevistado, 12) remetendo para uma lógica social de práticas de online dating.

Só após o match é que os utilizadores podem trocar mensagens entre si. A maioria dos entrevistados $(n=14)$ referiram que na maior parte das vezes tomavam a iniciativa, sendo que essa primeira abordagem tinha como ponto de partida informações que encontravam nos perfis.

Eu quando faço o match e quando há uma descrição etc. aquilo que eu tento fazer é arranjar pontos de ligação comigo e pegar por aí ou seja, aquele olá tudo bem e depois a conversa morre não tem piada. Eu tento entrar a matar entre aspas, pegar nalguma coisa que eu identifique que eu sei e me sinta confortável e entrar por aí (Entrevistado, 13).

Todavia, o facto de existir um match e de ser desbloqueada a função chat, não garantia a existência de comunicação ou caso esta existisse que fosse considerada interessante, gerando alguma frustração, resultando em que os utilizadores deixassem de comunicar entre si.

O conteúdo das mensagens também está sujeito a regras. É desincentivado o envio de conteúdo inapropriado, desrespeitoso ou ofensivo, alertando que quem o faça poderá ser banido e incitando novamente os utilizadores a denunciar tal situação, porém são inúmeros os relatos de conteúdos inadequados que são trocados (Thompson, 2018). Um entrevistado relatou ter sido alvo de um match de ódio, porém apenas fez unmatch e não denunciou o perfil.

Apesar da existência de um sistema de troca de mensagens no Tinder, um ponto comum entre todos os participantes do estudo $(n=18)$ foi o facto de estes referirem a migração da conversa para outras plataformas como o WhatsApp, Facebook Messenger ou Instagram. Os argumentos apresentados relacionavam-se com questões de empatia, de se sentirem confortáveis para trocar outro meio de contacto, com o facto de integrarem o contacto em outras ferramentas que já utilizavam, mas também aspetos relacionados com questões técnicas do Tinder como o facto de os participantes considerarem que o chat não funcionava corretamente, tendo alguns bugs tais como não receberem as mensagens de forma imediata, não conseguirem consultar corretamente o fluxo de troca de mensagens ou de limitações impostas como a impossibilidade de partilhar um link ou enviar uma fotografia estando a conversação limitada a texto, emojis e GIFs. Um dos participantes considerou ser uma vantagem não poderem ser trocadas imagens através do chat evitando receber fotos de pessoas nuas. 
Esta é uma forma clara de como os utilizadores contornam o funcionamento da aplicação ainda que esta tente condicionar a utilização, mas também de como a comunicação através de plataformas de troca instantânea de mensagens está socialmente instituída.

\section{Considerações finais}

Os utilizadores são o meio principal através dos quais as plataformas obtêm informações e onde, através das ações destes, aprendem sobre que informação é considerada mais interessante e visionada. Paralelamente os utilizadores têm influência em como as plataformas são desenvolvidas e como funcionam, embora tenham pouca consciência sobre esse facto, sendo a ação e consequente influência do algoritmo ignorada ou esquecida pela maioria dos entrevistados $(\mathrm{n}=11)$.

Aquando da criação do perfil, e devido à possibilidade de o fazer desde o Facebook, os utilizadores apesar de compreenderem a importância do mesmo, tendem a não prestar grande atenção sendo raro, na sua maioria, alterarem fotografias ou atualizarem a informação ou quando o fazem é de forma pontual.

As regras impostas pela aplicação condicionam as ações dos utilizadores e limitam as suas experiências de uso. Porém, parte destas são passíveis de ultrapassar após um pagamento o que nos leva a afirmar que o Tinder, limitando o número de swipes, localização, ou a permissão de retroceder ações, entre outras modalidades, frusta deliberadamente os utilizadores com o objetivo de os tentar converter em pagadores. Esta frustração leva a períodos de não utilização e como forma de manter os seus utilizadores motivados e ativos, e dos seus algoritmos continuarem a aprender, e com um objetivo de conversão, o Tinder envia notificações a incentivar a utilização indicando, por exemplo que existem matches à espera ou que o perfil está a quase a desaparecer acompanhado com a ilustração de um fantasma.

Foi percetível, através do discurso dos entrevistados, que estes não se questionavam sobre o porquê de lhes serem mostrados determinados perfis e não outros, ignorando o peso da ação de cada swipe e lamentando-se sobre um número cada vez menor de matches comparativamente a períodos anteriores. Tendo em conta que só através do match é possível iniciar uma conversa, podemos afirmar que o Tinder condiciona o número de matches de forma a limitar as possibilidades de cada utilizador.

Como consequência proporcionam novas lógicas de funcionamento levando a que, por vezes, os utilizadores contornem as restrições ou imposições de forma criativa. É exemplo disso o recurso a outos serviços de mensagem para comunicar visto o chat do Tinder restringir o formato da comunicação.

A própria interface do Tinder, o seu design e consequentemente o significado e poder atribuído simbolicamente a cada uma das suas opções pelos seus utilizadores, confere-lhes uma função de curador, mas sempre condicionados por terceiros: os proprietários, os programadores e os algoritmos, e remodelando as dinâmicas instituídas com base no espaço onde a interação tem lugar (De Souza e Silva e Frith, 2012).

Os algoritmos são parte estrutural e estruturante das soluções de online dating, porém a ausência de informação por parte das plataformas sobre o seu desenho, para que estão destinados, com base em que informações específicas e dados de uso vão aprendendo e como as ações dos utilizadores contribuem para o seu funcionamento 
e são, de forma dialética, moldadas, resultam numa dificuldade acrescida na investigação. Não obstante esta opacidade, é possível afirmar que a compreensão da lógica de funcionamento das aplicações é fundamental no sentido de perceber como estas, seja através do design ou através do discurso, contribuem estruturando atitudes e práticas sociais.

\section{Agradecimentos}

O nosso agradecimento a José Moreno, ISCTE-IUL, pelos inputs gerados após leitura preliminar.

\section{Referências}

Albury, K., Burgess, J., Light, B., Race, K. e Wilken, R. (2017). Data cultures of mobile dating and hook-up apps: Emerging issues for critical social science research. Big Data \& Society, 1-11.

Alpaydin, E. (2014). Introduction to machine learning. Cambridge, MA: MIT press.

Apresentamos o smarth photos para que você tenha mais deslizadas (2016, 13 de outubro). Disponível em https://blog.gotinder.com/introducing-smart-photos-for-the-mostswipeworthy-you/ [2018, 29 de junho]

Blackwell, C., Birnholtz, J. e Abbott, C. (2015). Seeing and being seen: Co-situation and impression formation using Grindr, a location-aware gay dating app. New Media \& Society, 17(7), 1117-1136.

Bryman, A. (2012). Social research methods. Oxford: Oxford University Press.

Carr, A. (2016, 1 de novembro). I found out my secret internal Tinder rating and now I wish I hadn't. Disponível em: www.fastcompany.com/3054871/whats-your-tinder-scoreinside-the-apps-internal-ranking-system [2018, 29 de junho]

Casimiro, C. (2014). Portuguese online dating: exploring gender differences in selfpresentations. Revista Teknokultura, 11(1), 117-141.

Chan, L. S. (2017). Who uses dating apps? Exploring the relationships among trust, sensation seeking, smartphone use, and the intent to use dating apps based on the Integrative Model. Computers in Human Behavior, 72, 246-258.

Choi, EP-H., Wong, JY-H., Lo, HH-M., Wong, W., Chio, JH-M. e Fong, DY-T. (2016). The Impacts of Using Smartphone Dating Applications on Sexual Risk Behaviours in College Students in Hong Kong. PloS ONE, 11(11).

Community guidelines (n.d.). Disponível em: www.gotinder.com/community-guidelines [2018, 29 de junho]

Courtois, C. e Timmermans. E. (2018). Cracking the Tinder code: An Experience Sampling Approach to the Dynamics and Impact of Platform Governing Algorithms. Journal of Computer-Mediated Communication, 23(1), 1-6.

David, G. e Cambre, C. (2016). Screened Intimacies: Tinder and the Swipe Logic. Social Media + Society, 2(2),1-11.

De Souza e Silva, A. e Frith, J. (2010). Locative Mobile Social Networks: Mapping Communication and Location in Urban Spaces, Mobilities, 5(4), 485-505.

Digital Advertising for Marketers, Agencies and Brancs. (n.d.) Disponível em www. matchmediagroup.com [2018, 31 de maio]

Domingos, P. (2017). The master algorithm. London: Penguin. 
Duguay, S. (2017). Dressing up Tinderella: interrogating authenticity claims on the mobile dating app Tinder. Information, Communication \& Society, 20(3), 351-367.

Ellison, N.B., Heino, R. D. e Gibbs, J. L. (2006). Managing Impressions online: Selfpresentation in the Online Dating Environment. Journal of Computer-Mediated Communication, 11, 415-441.

Gibbs, J. L., Ellison, N. B. e Heino, R. D., (2006). Self-Presentation in Online Personals The Role of Anticipated Future Interaction, Self-Disclosure, and Perceived Success in Internet Dating. Communications Research, Sage Publications, 33 (2), 152-177.

Gibson, J. J. (1979/1986). The ecological approach to perception. Hillsdale, NJ: Lawrence Erlbaum Associates.

Gillespie, T. (2014). The relevance of algorithms. Em T. Gillespie, P. Boczkowski e K. Foot, (Eds) Media technologies: essays on communication, materiality, and society, 167-194. Cambridge, Mass: MIT Press.

Gillespie, T. (2015). Platforms Intervene. Social Media + Society, 1(1), 1-2.

Gillespie, T. (2017). Governance of and by platforms. Em J. Burgess, A. Marwick e T. Poell (Eds) SAGE Handbook of Social Media, 1-30. London: SAGE.

Goluboff, S. (2015). Text to sex: The impact of cell phones on hooking up and sexuality on campus, Mobile Media \& Communication, 4(1), 102-120.

Guadagno, R., Oldie, B., Kruse, S. (2012). "Dating deception: Gender, online dating, and exaggerated self-presentation", Computers in Human Behavior, 22, 642-64.

Hjorth, L., e Lim, S.S. (2012). Mobile intimacy in an age of affective mobile media. Feminist Media Studies, 12(2), 477-484.

Hogan, B. (2010). The Presentation of Self in the Age of Social Media: Distinguishing Performances and Exhibitions Online. Bulletin of Science, Technology \& Society, 30, 377 386.

Krippendorff, K. H. (2012). Content Analysis: An Introduction to Its Methodology, $3 a$. ed. Thousand Oaks: Sage

LeFebvre, L. (2017). Swiping me off my feet: Explicating relationship initiation on Tinder. Journal of Social and Personal Relationships, 35(9) 1205-1229.

Licoppe, C., Riviére, C.A. e Morel, J. (2016). Grindr casual hook-ups as interactional achievements, New Media \& Society, 18(11), 2540-2558.

Light, B. (2014). Disconnecting with social networking sites. Basingstoke: Palgrave Mcmillan.

Light, B., Burgess, J., e Duguay, S. (2018). The walkthrough method: An approach to the study of apps. New media \& society, 20(3), 881-900.

Lo Iacono, V., Symonds, P. e Brown, D. H.K. (2016). Skype as a Tool for Qualitative Research Interviews. Sociological Research Online, 21(2), 12.

Marcus, S. R. (2016, 9-13 de junho). "Swipe to the right": Assessing self-presentation in the context of mobile dating applications. Apresentado em Annual Conference of the International Communication Association (ICA), Fukuoka, Japan.

McGrath, F. (2015, 24 de abril). What to know about Tinder in 5 charts. Disponível em: https://blog.globalwebindex.com/trends/what-to-know-about-tinder/ [2018, 31 de maio]

O’Brien, S. A. (2015, 3 de março). Tinder angers swipe-happy users. Disponível em: http:// money.cnn.com/2015/03/03/technology/tinder-plus/index.html [2018, 29 de junho]

Online dating - Statistics \& Facts (n.d.) Disponível em: www.statista.com/topics/2158/ online-dating [2018, 31 de julho]

Prigg, M. (2015, 15 de abril). Swipe right for puppy pictures. Tinder teams up with Instagram to allows users to show off their photos on their profiles. Daily Mail. Disponível em: 
www.dailymail.co.uk/sciencetech/article-3040649/Swipe-right-puppy-pictures-Tinderteams-Instagram-allows-users-photos.html [2018, 31 de maio]

Ranzini, G. e Lutz, C. (2017). Love at first swipe? Explaining Tinder self-presentation and motives. Mobile Media \& Communication, 5(1), 80-101.

Salmons, J. E. (2016). Doing Qualitative Research Online, London, Sage.

Schiling, M. (n.d.). 10 ways to catch out a catfish. Disponível em: www.eharmony.com.au/ dating-advice/trust-and-safety/10-ways-to-catch-out-a-catfish [2018, 21 de julho]

Schrock, A. (2015). Communicative affordances of mobile media: portability, availability, locatability, and multimediality. International Journal of Communication, 9, 1229-1246.

Sepúlveda, R. e Vieira, J. (2018). A aplicação de online dating Tinder na imprensa portuguesa: uma análise exploratória entre 2012-2017. Em Ibercom. XV Congresso Ibercom (Comp.) (pp. 2177 - 2206). Lisboa: Assibercom.

Sumter, S., Vandenbosch, L., e Ligtenberg, L. (2016). Love me Tinder: Untangling emerging adults' motivations for using the dating application Tinder. Telematics and Informatics, 34 (1), 67-78.

Thompson, L. (2018). I can be your Tinder Nightmare: Harassment and misogyny in the online sexual marketplace. Feminism \& Psychology, 28(1), 69-89.

Timmermans, E., e De Caluwé, E. (2017). Development and validation of the Tinder motives Scale (TMS). Computers in Human Behavior, 70, 341-350.

van Dijck, J. (2013). The culture of connectivity: A critical history of social media. Oxford, UK: Oxford University Press

Vieira, J. e Sepúlveda, R. (2017). A autoapresentação dos portugueses na plataforma de online dating Tinder. Observatorio (OBS*), 11(3), 153-185.

Ward, J. (2016). What are you doing on Tinder? Impression management on a matchmaking mobile app. Information, Communication \& Society, 20 (11), 1644-1659.

What is the Tinder Swipe Limit (Per 12 Hours)? Is It Lower for 2018? (2018, 10 de março). Disponível em: https://blog.photofeeler.com/tinder-swipe-limit/ [2018, 29 de junho].

What is Tinder (n.d). Disponível em: www.help.tinder.com/hc/en-us/articles/115004647686What-is-Tinder- [2018, 29 de junho].

Yeo, T. E. D. e Fung, T. H. (2017). "Mr Right Now": Temporality of relationship formation on gay mobile dating apps. Mobile Media \& Communication, 6, 3-18. 